\title{
Examining Collective Memory Building in Wikipedia: A Multilevel Network Approach
}

\author{
$\mathrm{Yu} \mathrm{Xu}$ \\ Annenberg School for \\ Communication and Journalism \\ University of Southern California \\ xuyu@usc.edu
}

\author{
Yusi Liu \\ College of Media and International \\ Culture \\ Zhejiang University \\ liuyusi@zju.edu.cn
}

\author{
Li Qi \\ College of Media and International \\ Culture \\ Zhejiang University \\ qili1013@163.com
}

\begin{abstract}
This study interprets Wikipedia as a memory place where independent contributors discuss and negotiate the meanings of past events in a collaborative way. We examine how interconnections between high-impact events lead to the differential patterns of collective memory building. The results show that the presence of a direct network tie between two events is related to a smaller difference in the patterns of collective memory building among Wikipedia users. In addition, a higher degree of structural equivalence between two events makes them more similar in terms of the patterns of collective memory building. Our findings reveal that the range of network effects is not confined within the local substructure but can extend to the global level as well. The results also confirm that Wikipedia editors will refer to the editing patterns of the events that have direct links or occupy similar structural positions with those they intend to contribute to.
\end{abstract}

\section{Introduction}

The term "collective memory" was first introduced by Halbwachs [1] and has been widely used in many disciplines in humanities and social sciences. Collective memory is generally defined as "a continuous, active process of sense-making and negotiation between past and present" [2]. Memory is collective because it is shared and used by a group delimited in time and space [3]. Collective memory is socially constructed in the sense that remembering is situated in a given socio-cultural context [1, 4]. Therefore, not only does collective memory shape the remembrance of the past but also it reflects how individuals construe current issues [5]. Analyzing how events are remembered is essential because a common image of the past strengthens collective identity among group members and provides guidelines for future behavior $[1,6]$.
The expansion of various kinds of new information and communication technologies has offered a unique opportunity for researchers to examine collective memory. Prior research has demonstrated that digital media can mediate collective memory processes as well as create a new form of memory production $[7,8]$. For example, Pentzold [9] has argued that Wikipedia is a global memory place where participants can provide and debate divergent interpretations of the past, resulting in "the formation and ratifications of shared knowledge that constitutes collective memory" [9]. This platform is global because it is open to anyone who has access to the Internet and is thus not constrained by national boundaries. For example, editors of the Chinese version of Wikipedia can be any online user who understands the Chinese language. The site is conceived as a memory place in the sense that it enables contributors from heterogeneous backgrounds to participate in "an intense process of discursive knowledge constitution" [9].

Following this argument, this research interprets Wikipedia as an online platform where collective memories about high-impact events are built in a collaborative manner. The objective of this study is to look at the mechanism that drives the differential patterns of collective memory building between significant Chinese events in Wikipedia. Although collective memories serve as a more important directive function in Chinese culture than in others [10], it is surprising that no prior study has been conducted to investigate the patterns of digital collective memory building in the context of China. This issue is worthy of investigation because it provides new insights into how collective memory is constructed and negotiated, considering that Wikipedia is an emerging place for collective memory building. In addition, examining this phenomenon is an important first step in evaluating how digital collective memories shape Chinese value systems and direct behavior in the future. 


\section{Contributions}

This paper makes several contributions to the extant literature. First, given the under-presentation of China in the literature on digital collective memory building, this study contributes to our understanding of the ways in which Wikipedia users construct and negotiate meanings of past events and the dynamics affecting this process. It opens the gate for future research in this important but largely overlooked area. Second, this work, to the best of our knowledge, is the first to take a multilevel network approach to investigating digital collective memories. Our empirical findings demonstrate that the range of network effects varies from the micro, local level to the macro, global level. Thirdly, we contribute to the promotion of multilevel hypothesis testing in network research. Although taking a network perspective enables us to collect data from multiple levels of analysis, prior research is limited in analyzing multilevel network data separately, which precludes "direct comparisons of theoretical influences at different levels" [11].

\section{Related work}

There are three streams of literature related to our studies: studies of collective memory building in Wikipedia; studies that examine cooperative work in Wikipedia from a network perspective; studies of the factors that influence users' contributions to Wikipedia.

Launched in 2001, Wikipedia is a free online encyclopedia written by millions of voluntary users. Researchers have stressed that Wikipedia offers powerful representations of the past and shapes a new digital memory culture where "memories are not anymore just consumed but also creatively produced in a participatory and decentralized way" [8]. Wikipedia also provides behavioral guidelines for adding and editing contents, which influences how contributors write articles. The most significant rule is the Neutral Point of View (NPOV). According to Wikipedia policies, NPOV refers to "representing fairly, proportionately, and as far as possible without bias, all significant views that have been published by reliable sources" [12].

As the construction of collective memories in Wikipedia can be easily observed by checking the editing history of an article [6], several empirical studies have directly investigated this phenomenon. For example, Aragon and his colleagues [13] conducted a cross-cultural study of network links between biographical articles in Wikipedia. The finding revealed great similarities between Wikipedias from different language versions, especially for geographically and linguistically closer communities. Ferron and Massa [6] showed that affective, cognitive, and social processes underlying Wikipedia representation of man-made and natural traumatic events were significantly different. In another study, they analyzed commemoration activities of traumatic events in Wikipedia and provided empirical evidence that a high volume of editing activities that occurred during anniversaries was linked to traumatic events rather than to non-traumatic events [8]. Kanhabua and his colleagues [3] examined the factors that triggered collective memories in Wikipedia. Their analysis confirmed that, besides location and temporal proximity, shared classification between events was also a determinant of which events were remembered. However, there is still one limitation in the extant literature. Despite the importance of collective memories in Chinese culture [10], what remains unknown is how collective memories about highimpact Chinese events are formed and constructed in Wikipedia.

Researchers have recognized that it is promising to adopt a network perspective to analyze digital collective memories, and Wikipedia provides an excellent data source for network analysis. For example, Ferron and Massa [14] conducted a network analysis of communication interactions in the talk page of the "2011 Egyptian revolution." Their research demonstrated the utility of the editing history in extracting various networks. However, what is still lacking is a conceptual framework that explains the intricate network dynamics affecting collective memory processes in Wikipedia. The present study tries to address this knowledge gap by applying network theories to the study of collective memory building.

Wikipedia has already been studied from a network perspective. For example, the structure of interactions in the user talk page network helped to distinguish between substantive experts and technical editors [15]. Moreover, preexisting social capital, operationalized as the proportion of collaborators being members of a pre-existing network and the overall cohesiveness of a pre-existing network, increased the efficiency of collaboration among Wikipedia contributors [16]. A recent study further suggested that an intermediate level of cohesion in the collaboration network improved WikiProject efficiency [17].

As an online encyclopedia created by peer production, Wikipedia largely depends on users who volunteer their time, skills, and expertise to enrich its content. Thus, numerous studies have explored the factors that influence individual contributions to the platform. Prior research has shown that demographic 
and socioeconomic characteristics are significant predictors of gaps in editing in Wikipedia. Particularly, the most likely contributors are males with high Internet skills [18]. As for psychological factors, Wikipedia contributors are largely driven by selfconcept motivation [19], altruistic motivations [20], desires to learn [21], gratification obtained from the engagement [22], and a sense of belonging to the Wikipedia community [23]. In addition, features of articles also play an important role in determining contribution patterns. Wikipedia users are more likely to edit in breaking news articles than in contemporaneous and historical articles [24]. While many demographic and socioeconomic backgrounds, psychological characteristics, and article attributes potentially influence the editing pattern, in this paper we are interested in how connections between events affect Wikipedia users' engagement in collective memory building.

\section{Hypotheses}

This study extends network theories to examine the patterns of collective memory building in Wikipedia. Homophily as a function of network embeddedness is one theoretical interest in network research $[25,26]$. The theory of homophily indicates that the emergence of a relationship is based on the similarities of socioeconomic, behavioral, and intrapersonal characteristics between social actors [11]. Two explanatory mechanisms help explain how networks influence homophily in actor behavior: the connectionist and the structuralist [25]. The connectionist approach proposes that actor behavior is an outcome of an information transmission process through direct relationships. Events that are directly connected with each other tend to share similar patterns of collective memory building in Wikipedia. It can be inferred that the presence of a dyadic tie at a micro level of analysis is likely to increase mutual awareness of Wikipedia contributors in two related events, thus shaping behaviors of contributors in the direction of making the editing patterns more homogenous.

H1: The presence of a direct network tie between two events is related to a smaller difference in the patterns of collective memory building among Wikipedia users.

Following the proposition of the connectionist approach, the meso level of analysis in this study operates at the clique level. By definition, a clique "consists of a subset of nodes, all of which are adjacent to each other" [27]. As a clique should be composed of three or more nodes in the network, dyads are not interpreted as cliques. Previous studies have shown that actors behave similarly to the extent they are comembers of multiple cliques [26, 28]. As the overlap between network actors increases, cohesive in-group members are more likely to imitate each other and consequently share similar characteristics. Thus, we formulate the following prediction:

H2: The intensity of clique co-membership between two events is related to a smaller difference in the patterns of collective memory building among Wikipedia users.

The structuralist approach proposes that actors occupying similar positions will likely have similar behavioral patterns, even though they are not directly connected with each other. Network scholars holding this view generally conceptualize the similar network environments that yield homophily as structural equivalents [29]. Structural equivalence is defined as "the degree two individuals have the same relations with the same others" [30], regardless of whether there is a direct link between them. Considerable empirical evidence from prior studies suggests that homophily of behaviors results from structural equivalence [25, 30]. It is expected that Wikipedia users will refer to the editing patterns of the events that are inherently similar to the ones they intend to contribute to. In view of this, the present study hypothesizes that the patterns of collective memory building between two high-impact events may become similar because both of them share similar network positions.

H3: The degree of structural equivalence between two events is related to a smaller difference in the patterns of collective memory building among Wikipedia users.

\section{Methods}

\subsection{Sample}

The present study chooses high-impact events listed on the page of "History of the People's Republic of China" in the Chinese version of Wikipedia. Eventually, researchers manually recorded all 94 mentioned events and their respective articles in Wikipedia on August 15, 2012. These events include the Cultural Revolution, the Great Tangshan Earthquake, the Sino-Vietnamese War, Hong Kong Reunification, the 2008 Summer Olympics in Beijing, the 2011 Wenzhou Train Collision, and so on.

\subsection{Measures}

5.2.1. Dependent variables. This paper argues that the access to the revision history of an article is helpful to 
examine the formation and transformation of collective memories [3]. The dependent variable is the difference in the patterns of collective memory building between a pair of events. This variable was operationalized as the absolute difference in the proportion of major/minor edits between two events. Contributors can choose to make either a major or a minor change to an article. The distinction between major and minor edits is important because a major change is disputable and affects the meaning of an article, and the modifications to the content need to be checked by other page editors. By contrast, a minor edit is one that the editors believe that no review is required for its acceptability. Typical minor edits include correcting spelling and grammatical errors, making simple formatting and layout changes, fixing broken external links and references, and adding internal links. Registered Wikipedia users can mark edits as minor when saving a revised page. Marking a major change as a minor one is not conceived as good etiquette in the community of Wikipedia. Calculating the proportion of major/minor edits is a good measure of the patterns of collective memory building in that it reflects the degree of conformity of a particular event among Wikipedia contributors. It is also a better measure than the total number of major/minor edits because larger articles are more likely to receive more edits from independent contributors.

The editing history of each Wikipedia article provides detailed information about edits per year and user statistics [6]. Based on this information, the research assistant recorded the proportion of major/minor edits for each event within a three-year window (from 2013 to 2015). The dependent variable was then computed based on this information.

5.2.2. Independent variables. This study relies on the mention network of significant events in the history of China to compute three major independent variables. The researchers ran a Python program to collect the network data on August 19, 2012. One number (1 or 0) was assigned for each pair of events depending on whether the Wikipedia page of event $i$ mentioned the name of event $j$. A binary, directional, asymmetrical matrix of events was thus constructed. A visualization of this network is presented in Figure 1. The size of each node is proportional to the number of received links. An edge represents whether there was a link from event $i$ to event $j$.

The local level variable is a direct network tie, which refers to the presence or absence of a network link between each pair of nodes.

The intermediate level variable is clique comembership. To measure this variable, the researchers used UCINET to identify all the cliques within this network. Co-membership was thus computed by counting the total number of cliques where two events were joint members.

The global level variable is structural equivalence. It was measured by Jaccard coefficient. Jaccard coefficient is a representative measurement method among various similarity indexes. It counts the proportion of the times that two nodes are linked to the same alter [13].

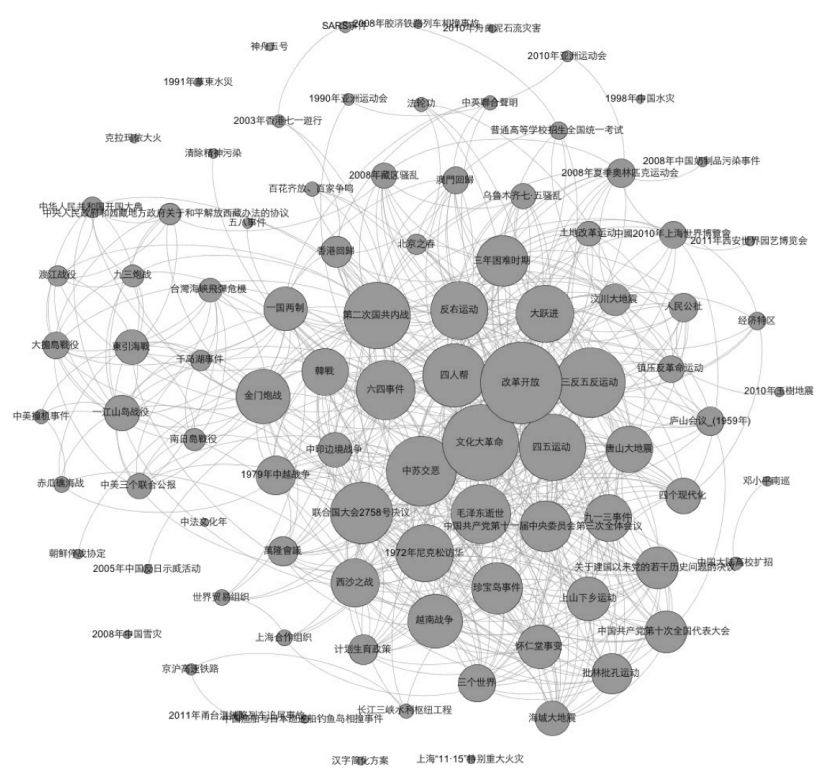

Figure 1. A visualization of the mention network of significant events in the history of China using Gephi

5.2.3.Control variables. Prior research has shown that other event-related attributes explain variation in the patterns of collective memory building among Wikipedia users [8, 24]. In view of this, the researchers recorded the start year of an event, the duration of an event, and the year of the first edit in Wikipedia. Two coders then employed the codebook to determine whether it was a national or local event (Krippendorff's $\alpha=.97$ ), and whether it was a traumatic or non-traumatic event (Krippendorff's $\alpha=.89$ ).

Table 1. Descriptive statistics of the key variables

\begin{tabular}{|l|c|c|c|c|c|}
\hline & $\mathrm{N}$ & Min & Max & $\mathrm{M}(\%)$ & $\mathrm{SD}$ \\
\hline $\begin{array}{l}\text { Node } \\
\text { attributes }\end{array}$ & & 29 & 100 & 71.86 & 12.39 \\
\hline $\begin{array}{l}\text { The } \\
\text { proportio } \\
\text { n of major }\end{array}$ & 94 & 29 & & \\
\hline
\end{tabular}




\begin{tabular}{|c|c|c|c|c|c|}
\hline $\begin{array}{l}\text { edits } \times \\
100\end{array}$ & & & & & \\
\hline Start year & 94 & 1943 & 2007 & $\begin{array}{c}1976.4 \\
8\end{array}$ & 20.69 \\
\hline $\begin{array}{l}\text { Duration } \\
\text { (days) }\end{array}$ & 94 & 1 & $\begin{array}{c}1503 \\
5\end{array}$ & $\begin{array}{c}1192.7 \\
8\end{array}$ & $\begin{array}{c}2842 . \\
23\end{array}$ \\
\hline $\begin{array}{l}\text { Year of } \\
\text { the first } \\
\text { edit }\end{array}$ & 94 & 2003 & 2012 & $\begin{array}{c}2005.8 \\
7\end{array}$ & 2.31 \\
\hline $\begin{array}{l}\text { A national } \\
\text { event }\end{array}$ & 94 & 0 & 1 & $62 \%$ & \\
\hline $\begin{array}{l}\text { A } \\
\text { traumatic } \\
\text { event }\end{array}$ & 94 & 0 & 1 & $21 \%$ & \\
\hline $\begin{array}{l}\text { Dyadic } \\
\text { attributes }\end{array}$ & & & & & \\
\hline $\begin{array}{l}\text { The } \\
\text { proportio } \\
\mathrm{n} \text { of } \\
\text { major/min } \\
\text { or edits } \times \\
100 \text { (diff.) }\end{array}$ & 8742 & 0 & 71.43 & 13.60 & 11.05 \\
\hline $\begin{array}{l}\text { Direct } \\
\text { network } \\
\text { tie }\end{array}$ & 8742 & 0 & 1 & $11 \%$ & \\
\hline $\begin{array}{l}\text { Clique co- } \\
\text { membersh } \\
\text { ip }\end{array}$ & 8742 & 0 & 17 & .21 & 1.08 \\
\hline $\begin{array}{l}\text { Structural } \\
\text { equivalen } \\
\text { ce }\end{array}$ & 8742 & 0 & 1 & .11 & .17 \\
\hline $\begin{array}{l}\text { Start year } \\
\text { (diff.) }\end{array}$ & 8742 & 0 & 64 & 23.90 & 16.89 \\
\hline $\begin{array}{l}\text { Duration } \\
\text { (diff.) }\end{array}$ & 8742 & 0 & $\begin{array}{c}1503 \\
4\end{array}$ & $\begin{array}{c}2040.9 \\
3\end{array}$ & $\begin{array}{c}3463 . \\
03\end{array}$ \\
\hline $\begin{array}{l}\text { Year of } \\
\text { the first } \\
\text { edit (diff.) }\end{array}$ & 8742 & 0 & 9 & 2.56 & 2.03 \\
\hline $\begin{array}{l}\text { A national } \\
\text { or local } \\
\text { event } \\
\text { (match) }\end{array}$ & 8742 & 0 & 1 & $52 \%$ & \\
\hline $\begin{array}{l}\text { A } \\
\text { traumatic } \\
\text { or non- } \\
\text { traumatic } \\
\text { event } \\
\text { (match) } \\
\end{array}$ & 8742 & 0 & 1 & $67 \%$ & \\
\hline
\end{tabular}

\subsection{Analytical procedures}

Since this study focuses on the similarity of collective memory building between events, the most appropriate unit of analysis should be dyads. But dyadic data are usually interdependent, thus violating the assumption of independent observations in OLS regression. In view of this, the present study chooses mixed-effects models that address non-independence between cases [31]. Mixed-effects models take into consideration the group contexts where individual exist. For model specifications, our dependent variable and control variables represent the absolute difference or match within each dyad (see Table 1 for details).

\section{Results}

The final results of mixed-effects models are presented in Table 2. Model 1 shows an intercept-only model without any predicting variable. This null model decomposed the variance in the dependent variable resulting from the non-independence of cases. The deviance score of the null model was used as the basis for evaluating the improvement in model fit in subsequent models. Chi-square tests were conducted to determine whether the reduction of deviance was significant. Model 2 estimates the effects of control variables. The full model (Model 3 in Table 2) further reveals how variables of theoretical interest influence the dependent variable, when other controlled variables are held constant. The results of the correlation matrix and covariance matrix for the fixed effects estimates show that there is no multicollinearity among predictors in both Model 2 and Model 3 (detailed results available on request).

As our dependent variable is the difference in the patterns of collective memory building between two events, a negative coefficient suggests that an increase of the predictor will reduce this gap. Hypothesis 1 predicted that the presence of a direct network tie between two events is related to a smaller difference in the patterns of collective memory building among Wikipedia users. The finding shows that direct network tie has a significant and positive effect, thus supporting H1 (Coef. $=-1.4099$, Std. Err. $=.4051, p$ $<.001)$. The presence of a direct network tie reduces the gap between two events by approximately $1.41 \%$. Hypothesis 2 predicted a negative relationship between the intensity of clique co-membership and the differential patterns of collective memory building among Wikipedia users. This hypothesis receives no empirical support because we find that clique comembership exerts no influence on the dependent variable (Coef. $=-.1192$, Std. Err. $=.1132, p=.293$ ). Hypothesis 3 predicted that a higher degree of structural equivalence between two events makes them more similar in terms of the patterns of collective memory building among Wikipedia users. Our result reveals that the effect of structural equivalence is 
significantly negative (Coef. $=-3.5774$, Std. Err. $=.7097, p<.001)$, which is consistent with the prediction of $\mathrm{H} 3$.

Table 2. Mixed-effects models of the similarity of collective memory building between events $(\mathrm{N}=8742)$

\begin{tabular}{|c|c|c|c|}
\hline & Model 1 & Model 2 & Model 3 \\
\hline & $\begin{array}{c}\text { Coef. (Std. } \\
\text { Err.) }\end{array}$ & $\begin{array}{l}\text { Coef. (Std. } \\
\text { Err.) }\end{array}$ & $\begin{array}{c}\text { Coef. (Std. } \\
\text { Err.) }\end{array}$ \\
\hline \multicolumn{4}{|l|}{$\begin{array}{l}\text { Fixed } \\
\text { effects }\end{array}$} \\
\hline \multirow[t]{2}{*}{ Intercept } & $\begin{array}{c}13.6048 * * \\
*\end{array}$ & $\begin{array}{c}12.7267 * * \\
*\end{array}$ & $\begin{array}{c}13.8223 * * \\
*\end{array}$ \\
\hline & $(.6109)$ & $(.6515)$ & $(.6658)$ \\
\hline \multirow[t]{2}{*}{$\begin{array}{l}\text { Start year } \\
\text { (diff.) }\end{array}$} & & .0022 & $-.0153 *$ \\
\hline & & $(.0062)$ & $(.0067)$ \\
\hline \multirow[t]{2}{*}{$\begin{array}{l}\text { Duration } \\
\text { (diff.) }\end{array}$} & & $-.0001 * *$ & $-.0001 *$ \\
\hline & & $(.0000)$ & $(.0000)$ \\
\hline \multirow[t]{2}{*}{$\begin{array}{l}\text { Year of the } \\
\text { first edit } \\
\text { (diff.) }\end{array}$} & & $.3195 * * *$ & $.2745 * * *$ \\
\hline & & $(.0561)$ & $(.0563)$ \\
\hline \multirow[t]{2}{*}{$\begin{array}{l}\text { A national } \\
\text { or local } \\
\text { event } \\
\text { (match) }\end{array}$} & & -.0044 & .0675 \\
\hline & & $(.2076)$ & $(.2082)$ \\
\hline \multirow[t]{2}{*}{$\begin{array}{l}\text { A traumatic } \\
\text { event } \\
\text { (match) }\end{array}$} & & $-.7680 * *$ & $-.7581 * *$ \\
\hline & & $(.2511)$ & $(.2505)$ \\
\hline \multirow[t]{2}{*}{$\begin{array}{l}\text { Direct } \\
\text { network tie }\end{array}$} & & & $-1.4099 * * *$ \\
\hline & & & $(.4051)$ \\
\hline \multirow[t]{2}{*}{$\begin{array}{l}\text { Clique co- } \\
\text { membership }\end{array}$} & & & -.1192 \\
\hline & & & $(.1132)$ \\
\hline \multirow[t]{2}{*}{$\begin{array}{l}\text { Structural } \\
\text { equivalence }\end{array}$} & & & $-3.5774 * * *$ \\
\hline & & & $(.7097)$ \\
\hline \multicolumn{4}{|l|}{$\begin{array}{l}\text { Random } \\
\text { effects }\end{array}$} \\
\hline$\sigma^{2}$ & 88.4158 & 87.8965 & 87.4341 \\
\hline$r_{0}$ & 34.1355 & 33.6084 & 33.0694 \\
\hline Deviance & 64328.144 & 64303.820 & 64255.346 \\
\hline $\begin{array}{l}\text { The } \\
\text { improvemen } \\
t \text { in model } \\
\text { fit }\end{array}$ & & Significant & Significant \\
\hline
\end{tabular}

\section{Discussion and conclusion}

This study interprets Wikipedia as a memory place where independent contributors collaboratively discuss and negotiate the meanings of past events. Building upon work of network scholars whose theoretical interest is to explain homophily as a function of network embeddedness, our research investigates how interconnections between high-impact events lead to the differential patterns of collective memory building in Wikipedia. It sheds light on how the remembrance of the past is socially constructed in the digital space. Despite the fact that collective memories play a more important directive role in Chinese culture than others, the process of digital collective memory building in the context of China is less understood and warrants attention from researchers. This work advances our understanding of this important yet largely overlooked phenomenon. Although the findings are not readily generalizable to other cultural contexts, the issues and possibilities discussed here are theoretically interesting and promising.

Our findings reveal that the range of network effects is not confined within the local substructure but can extend to the global level as well. Specifically, we provide empirical evidence that the presence of a direct network tie between two events is related to a smaller difference in the patterns of collective memory building among Wikipedia users. In addition, a higher degree of structural equivalence between two events makes them more similar in terms of the patterns of collective memory building. Inconsistent with our prediction, clique co-membership exerts no significant influence on this pattern, although the regression coefficient proves to be negative. These results confirm that interconnections between high-impact events facilitate imitation behavior, such that Wikipedia editors will refer to the editing patterns of the events that have direct links or occupy similar structural positions with those they plan to make a contribution to. It is argued that this imitation process is the main mechanism underlying the reduction of the differential editing patterns. The fact that clique comembership fails to explain this difference suggests one direction for future research about the range of network levels can operate. The lack of significance in the clique co-membership may be due to the fact that cliques are established based on direct ties. As the presence of a direct link explains more variance in the dependent variable, the coefficient of clique comembership becomes non-significant when this microlevel factor is controlled in the predicting model.

\section{Limitations and future directions}


Despite this study's contributions to the literature, it is still cannot be considered as comprehensive in its present form. There are several limitations that need to be addressed by future research. First, since our sample includes high-impact events in the history of China only, the generalizability of our conclusions may not be warranted without further testing. Prior research has already suggested that network effects may be contingent on the specific research context [26]. Second, this study fails to take into account more temporal micro-phenomena in the process of collective memory building. Apparently, the mention network of significant events may be changing over time because digital memories are continuously modified in Wikipedia. As unobtrusive and real-time data are recorded in the revision history of an article [2, 14], it is empirically possible for future researchers to rely on these large-scale datasets to conduct longitudinal analyses. Last but not least, this study neglects how the embeddedness of page editors explains the variation in the patterns of collective memory building between high-impact events. It is entirely possible that one contributor edits several related pages simultaneously. Future studies can take a multidimentional network approach to identify relationships among Wikipedia articles and contributors [24]. A two-mode network, consisting of Wikipedia pages of past events and individual contributors, can be constructed. Understanding co-editing patterns of Wikipedia users can also provide additional support about how far network effects can spill out.

\section{Acknowledgment}

This study was sponsored by Chinese National Social Science Foundation (15CXW030).

\section{References}

[1] Halbwachs, M. (1992). On Collective Memory. Chicago, IL: University of Chicago Press.

[2] Ferron, M., \& Massa, P. (2011). WikiRevolutions: Wikipedia as a lens for studying the real-time formation of collective memories of revolutions. International Journal of Communication, 5, 1313-1332.

[3] Kanhabua, N., Nguyen, T. B., \& Niederée, C. (2014). What triggers human remembering of events? A large-scale analysis of catalysts for collective memory in Wikipedia. In Proceedings of 14th ACM/IEEE-CS Joint Conference on Digital Libraries, 341-350. doi:10.1109/JCDL.2014.6970189

[4] Kligler-Vilenchik, N., Tsfati, Y., and Meyers, O. (2014). Setting the collective memory agenda: Examining mainstream media influence on individuals' perceptions of the past. Memory Studies, 7(4), 484-499. doi: $10.1177 / 1750698014523443$

[5] Olick, J. K., \& Levy, D. (1997). Collective memory and cultural constraint: Holocaust myth and rationality in German politics. American Sociological Review, 62(6), 921936. doi: $10.2307 / 2657347$

[6] Ferron, M., \& Massa, P. (2012). Psychological processes underlying Wikipedia representations of natural and manmade disasters. In Proceedings of the Eighth International Symposium on Wikis and Open Collaboration. doi:10.1145/2462932.2462935

[7] Wertsch, J. V. (2002). Voices of Collective Remembering. New York: Cambridge University Press.

[8] Ferron, M., \& Massa, P. (2014). Beyond the encyclopedia: Collective memories in Wikipedia. Memory Studies, 7(1), 22-45. doi:10.1177/1750698013490590

[9] Pentzold, C. (2009). Fixing the floating gap: the online encyclopedia Wikipedia as a global memory place. Memory Studies 2(2): 255-272. doi:10.1177/1750698008102055

[10] Wang, Q. (2008). On the cultural constitution of collective memory. Memory, 16(3), 305-317. doi: $10.1080 / 09658210701801467$

[11] Monge, P. R., \& Contractor, N. (2003). Theories of Communication Networks. New York: Oxford University Press.

[12] Wikipedia: The free encyclopedia. (2012). Wikipedia: Neutral point of view. Retrieved from http://en.wikipedia.org/wiki/Wikipedia:Neutral point of vie $\underline{\mathrm{w}}$

[13] Aragón, P., Laniado, D., Kaltenbrunner, A., \& Volkovich, Y. (2012). Biographical social networks on Wikipedia: a cross-cultural study of links that made history. In Proceedings of the Eighth Annual International Symposium on Wikis and Open Collaboration. doi: $10.1145 / 2462932.2462958$

[14] Ferron, M., \& Massa, P. (2011). Collective memory building in Wikipedia: the case of North African uprisings. In Proceedings of the 7th International Symposium on Wikis and Open Collaboration, 114-123. doi: $10.1145 / 2038558.2038578$

[15] Welser, H. T., Cosley, D., Kossinets, G., Lin, A., Dokshin, F., Gay, G., \& Smith, M. (2011). Finding social roles in Wikipedia. In Proceedings of the 2011 iConference, 122-129. doi:10.1145/1940761.1940778

[16] Nemoto, K., Gloor, P., \& Laubacher, R. (2011). Social capital increases efficiency of collaboration among Wikipedia editors. In Proceedings of the 22nd ACM 
conference on Hypertext and hypermedia, 231-240. doi: $10.1145 / 1995966.1995997$

[17] Qin, X., Cunningham, P., \& Salter-Townshend, M. (2015). The influence of network structures of Wikipedia discussion pages on the efficiency of WikiProjects. Social Networks, 43, 1-15. doi:10.1016/j.socnet.2015.04.002

[18] Hargittai, E. \& Shaw, A. (2015). Mind the Skills Gap: The Role of Internet Know-How and Gender in Contributions to Wikipedia. Information, Communication \& Society, 18(4), 424-442. doi: 10.1080/1369118X.2014.957711

[19] Yang, H. L., \& Lai, C. Y. (2010). Motivations of Wikipedia content contributors. Computers in Human Behavior, 26(6), 1377-1383. doi:10.1016/j.chb.2010.04.011

[20] Oreg, S., \& Nov, O. (2008). Exploring motivations for contributing to open source initiatives: The roles of contribution context and personal values. Computers in human behavior, 24(5), 2055-2073. doi:10.1016/j.chb.2007.09.007

[21] Baytiyeh, H., \& Pfaffman, J. (2010). Volunteers in Wikipedia: Why the community matters. Educational Technology \& Society, 13(2), 128-140.

[22] Schroer, J., \& Hertel, G. (2009). Voluntary engagement in an open web-based encyclopedia: Wikipedians and why they do it. Media Psychology, 12(1), 96-120. doi:10.1080/15213260802669466

[23] Cho, H., Chen, M., \& Chung, S. (2010). Testing an integrative theoretical model of knowledge-sharing behavior in the context of Wikipedia. Journal of the American Society for Information Science and Technology, 61(6), 1198-1212. doi: $10.1002 /$ asi.21316
[24] Keegan, B., Gergle, D., \& Contractor, N. (2012). Do editors or articles drive collaboration?: multilevel statistical network analysis of wikipedia coauthorship. In Proceedings of the ACM 2012 conference on computer supported cooperative work, 427-436. doi: 10.1145/2145204.2145271

[25] Borgatti, S. P., \& Foster, P. C. (2003) The network paradigm in organizational research: A review and typology. Journal of Management, 29(6), 991-1013. doi: 10.1016/S0149-2063_03_00087-4

[26] Pallotti, F., Tubaro, P., \& Lomi, A. (2015). How far do network effects spill over? Evidence from an empirical study of performance differentials in interorganizational networks. European Management Review, 12(3), 189-208. doi: $10.1111 /$ emre. 12052

[27] Wasserman, S., and Faust, K. (1994). Social Network Analysis: Methods and Applications. New York: Cambridge University Press.

[28] Rowley, T. J., Greve, H. R., Rao, H., Baum, J. A. C., \& Shipilov, A. V. (2005). Time to break up: Social and instrumental antecedents of firm exits from exchange cliques. Academy of Management Journal, 48(3), 499-520. doi: 10.5465/AMJ.2005.17407914

[29] Burt, R. (1992). Structural holes: The social structure of competition. Cambridge, MA: Harvard University Press.

[30] Valente, T. W. (1995). Network Models of the Diffusion of Innovations. Cresskill, NJ: Hampton Press.

[31] Valente, T. W. (2010). Social Networks and Health: Models, Methods, and Applications. New York: Oxford University

Press 